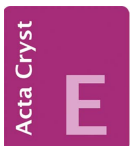

CRYSTALLOGRAPHIC COMMUNICATIONS

ISSN 2056-9890

Received 17 March 202

Accepted 2 April 2021

Edited by M. Weil, Vienna University of Technology, Austria

Keywords: crystal structure; cycloaddition product; 1,3,4,8-tetrahydro-2H-pyrido[1,2a]pyrimidine; Hirshfeld surface analysis.

CCDC reference: 2075706

Supporting information: this article has supporting information at journals.iucr.org/e

\section{Crystal structure and Hirshfeld surface analysis of 6-amino-8-(2,6-dichlorophenyl)-1,3,4,8-tetrahydro- 2H-pyrido[1,2-a]pyrimidine-7,9-dicarbonitrile}

Farid N. Naghiyev, ${ }^{a}$ Tatiana A. Tereshina, ${ }^{b}$ Victor N. Khrustalev, ${ }^{\text {b,c }}$ Mehmet

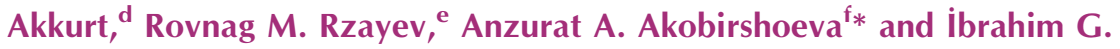
Mamedov ${ }^{\mathrm{a}}$

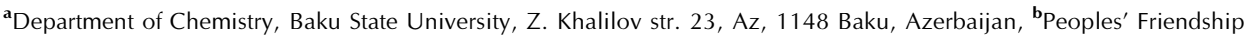
University of Russia (RUDN University), Miklukho-Maklay St. 6, Moscow, 117198, Russian Federation, '⿳N口. D. Zelinsky Institute of Organic Chemistry RAS, Leninsky Prosp. 47, Moscow, 119991, Russian Federation, d Department of Physics, Faculty of Sciences, Erciyes University, 38039 Kayseri, Turkey, "'Composite Materials" Scientific Research Center, Azerbaijan State Economic University (UNEC), H. Aliyev str. 135, Az 1063, Baku, Azerbaijan, and 'Acad. Sci. Republ. Tadzhikistan, Kh. Yu. Yusufbekov Pamir Biol. Inst., 1 Kholdorova St, Khorog 736002, Gbao, Tajikistan. *Correspondence e-mail: anzurat2003@mail.ru
\end{abstract}

In the molecular structure of the title compound, $\mathrm{C}_{16} \mathrm{H}_{13} \mathrm{Cl}_{2} \mathrm{~N}_{5}$, the 1,4dihydropyridine ring of the 1,3,4,8-tetrahydro- $2 H$-pyrido[1,2- $a$ ]pyrimidine ring system adopts a screw-boat conformation, while the 1,3-diazinane ring is puckered. In the crystal, intermolecular $\mathrm{N}-\mathrm{H} \cdots \mathrm{N}$ and $\mathrm{C}-\mathrm{H} \cdots \mathrm{N}$ hydrogen bonds form molecular sheets parallel to the (110) and (110) planes, crossing each other. Adjacent molecules are further linked by $\mathrm{C}-\mathrm{H} \cdots \pi$ interactions, which form zigzag chains propagating parallel to [100]. A Hirshfeld surface analysis indicates that the most significant contributions to the crystal packing are from $\mathrm{N} \cdots \mathrm{H} / \mathrm{H} \cdots \mathrm{N}(28.4 \%), \mathrm{H} \cdots \mathrm{H}(24.5 \%), \mathrm{C} \cdots \mathrm{H} / \mathrm{H} \cdots \mathrm{C}(21.4 \%)$ and $\mathrm{Cl} \cdots \mathrm{H} /$ $\mathrm{H} \cdot \mathrm{Cl}(16.1 \%)$ contacts.

\section{Chemical context}

Chemical transformations comprising carbon-carbon and carbon-heteroatom bond-formation reactions are fundamental tools in modern synthetic organic chemistry (Yadigarov et al., 2009; Abdelhamid et al., 2011; Khalilov et al., 2011; Yin et al., 2020). They are also used for the synthesis of valuable building blocks in medicinal chemistry, coordination chemistry and material science (Mahmoudi et al., 2017, 2019; Viswanathan et al., 2019).

Pyrido[1,2- $a$ ]pyrimidines constitute a valuable class of heterocycles because many of them possess broad biological activities, such as monoamine oxidase inhibition, antihypertensive, insecticide, serotonergic antagonist, analgesic, anti-inflammatory, cytoprotective, bronchodilatory, phosphodiesterase-inhibitory, antithrombotic, antiallergic, antiatherosclerotic and hypoglycaemic activities, as well as antitumor effects (Hermecz \& Mészáros, 1988; Ukrainets et al., 2018). The pyrido[1,2-a]pyrimidine motif is incorporated into the structure of some marketed drugs, including the antiasthmatic agent pemirolast, the tranquilizer pirenperone, the antiallergic agent ramastine, and the psychotropic agents risperidone and paliperidone (Awouters et al., 1986; Blaton et al., 1995; Yahata et al., 2006; Riva et al., 2011). Over recent decades, a number of synthetic protocols for the synthesis of pyrido[1,2-a]pyrimidines have been reported, and these 
approaches have focused on two-component reactions (Wu et al., 2003; Pryadeina et al., 2005). Multi-component reactions have developed as powerful tools for the design of complex molecules, natural products and drug-like molecules in a minimum number of synthetic steps (Abdelhamid et al., 2014; McLaughlin et al., 2014; Janssen et al., 2018).<smiles>N#CC1=C(N)N2CCCNC2=C(C#N)C1c1c(Cl)cccc1Cl</smiles>

As part of our studies on the chemistry of bridgehead nitrogen heterocycles, as well as taking into account our ongoing structural studies (Mamedov et al., 2013; Naghiyev et al., 2020a,b,c; Naghiyev et al., 2021), we report here the crystal structure and Hirshfeld surface analysis of the title compound, $\mathrm{C}_{16} \mathrm{H}_{13} \mathrm{Cl}_{2} \mathrm{~N}_{5}$, obtained by an efficient three-component synthetic protocol.

\section{Structural commentary}

In the molecular structure of the title compound, (Fig. 1), the 1,4-dihydropyridine ring (N5/C6-C9/C9A) of the 1,3,4,8tetrahydro- $2 H$-pyrido[1,2-a]pyrimidine ring system $(\mathrm{N} 1 / \mathrm{C} 2-$ C4/N5/C6-C9/C9A) adopts a screw-boat conformation with puckering parameters (Cremer \& Pople, 1975) $Q_{\mathrm{T}}=$ 0.520 (3) $\AA, \theta=120.8(3)^{\circ}$ and $\varphi=270.4(3)^{\circ}$, while the $1,3-$ diazinane ring $(\mathrm{N} 1 / \mathrm{C} 2-\mathrm{C} 4 / \mathrm{N} 5 / \mathrm{C} 9 A)$ is puckered $\left[Q_{\mathrm{T}}=\right.$ 0.160 (3) $\AA, \theta=75.2(11)^{\circ}$ and $\left.\varphi=169.4(10)^{\circ}\right]$. The dichlorophenyl ring (C11-C16) makes a dihedral angle of

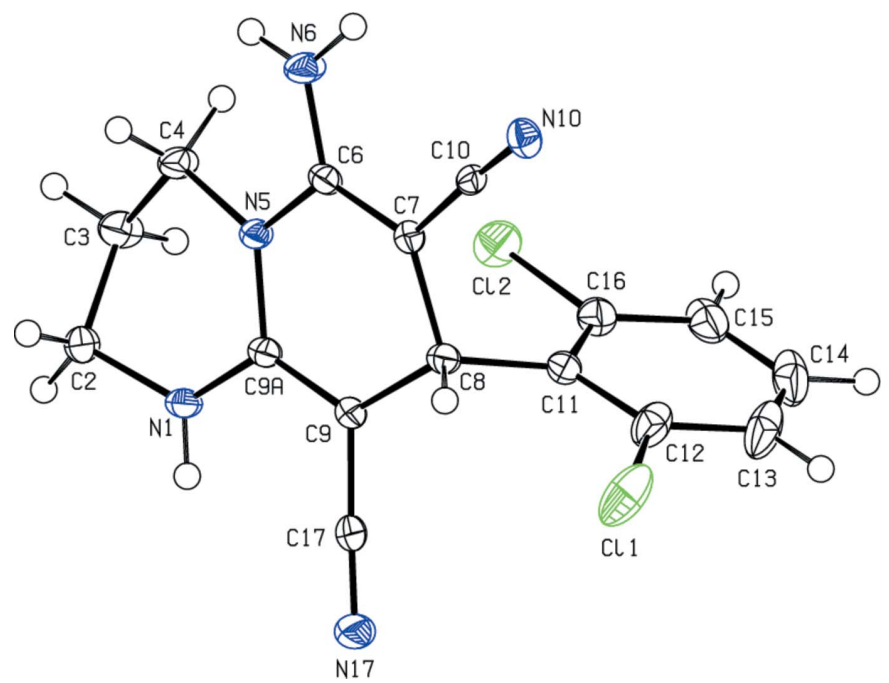

Figure 1

The molecular structure of the title compound. Displacement ellipsoids are drawn at the $50 \%$ probability level.

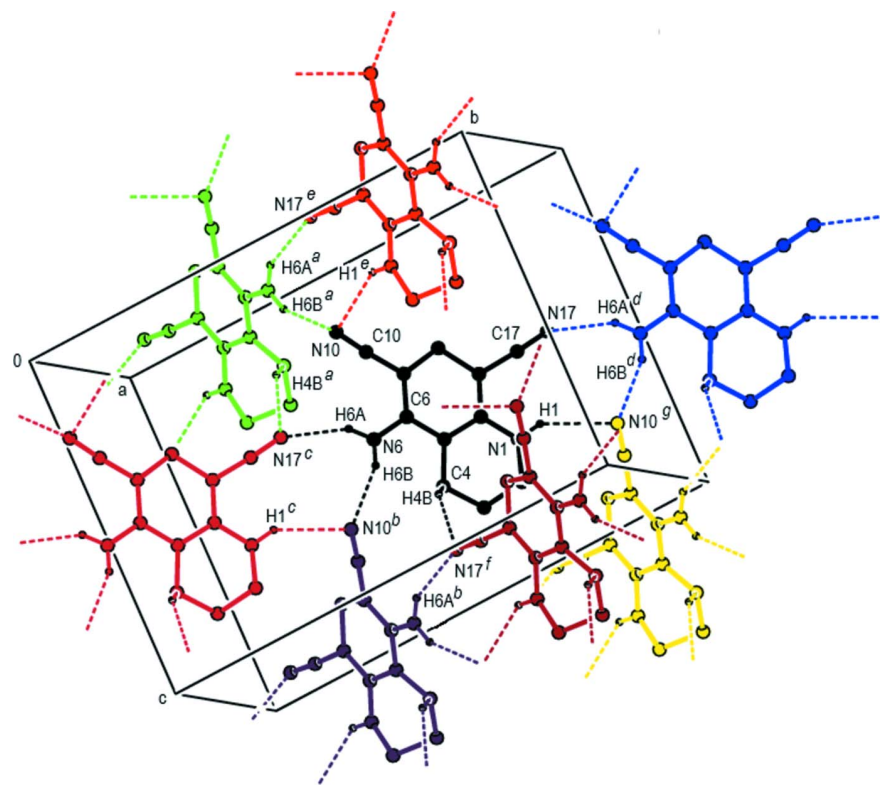

Figure 2

A view showing details of the intermolecular $\mathrm{N}-\mathrm{H} \cdots \mathrm{N}$ and $\mathrm{C}-\mathrm{H} \cdots \mathrm{N}$ hydrogen bonds in the unit cell of the title compound. The dichlorophenyl group and $\mathrm{H}$ atoms not involved in hydrogen bonding have been omitted for clarity. [Symmetry codes: (a) $x, 1-y,-\frac{1}{2}+z$; (b) $x, 1-y, \frac{1}{2}+z ;(c)$ $-\frac{1}{2}+x,-\frac{1}{2}+y, z ;(d) \frac{1}{2}+x, \frac{1}{2}+y, z ;(e)-\frac{1}{2}+x, \frac{3}{2}-y,-\frac{1}{2}+z ;(f)-\frac{1}{2}+x, \frac{3}{2}-y$, $\left.\frac{1}{2}+z ;(g) \frac{1}{2}+x, \frac{3}{2}-y, \frac{1}{2}+z\right]$.

$80.82(12)^{\circ}$ with the mean plane of the $1,3,4,8$-tetrahydro- $2 \mathrm{H}$ pyrido[1,2-a]pyrimidine ring system.

\section{Supramolecular features}

In the crystal, intermolecular $\mathrm{N}-\mathrm{H} \cdots \mathrm{N}$ hydrogen bonds between the amine functions as donor groups and the nitrile $\mathrm{N}$ atoms as acceptor groups and intermolecular $\mathrm{C}-\mathrm{H} \cdots \mathrm{N}$ hydrogen bonds lead to the formation of sheets extending parallel to (110) and (110) (Table 1; Figs. 2, 3 and 4). These hydrogen-bonded sheets cross each other (Fig. 5). C $-\mathrm{H} \cdots \pi$

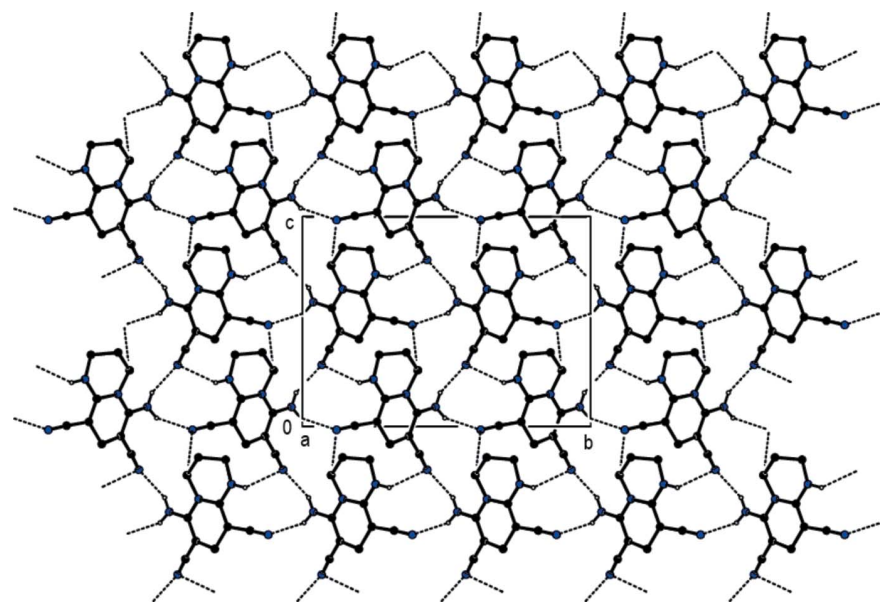

Figure 3

A view along [100] showing the intermolecular $\mathrm{N}-\mathrm{H} \cdots \mathrm{N}$ and $\mathrm{C}-\mathrm{H} \cdots \mathrm{N}$ hydrogen bonds of the title compound. The dichlorophenyl group and $\mathrm{H}$ atoms not involved in hydrogen bonding have been omitted for clarity. 
Table 1

Hydrogen-bond geometry $\left(\AA,^{\circ}\right)$.

$\mathrm{Cg} 3$ is the centroid of the C11-C16 dichlorophenyl ring.

\begin{tabular}{lllll}
\hline$D-\mathrm{H} \cdots A$ & $D-\mathrm{H}$ & $\mathrm{H} \cdots A$ & $D \cdots A$ & $D-\mathrm{H} \cdots A$ \\
\hline $\mathrm{N} 1-\mathrm{H} 1 \cdots \mathrm{N} 10^{\mathrm{i}}$ & $0.85(3)$ & $2.43(3)$ & $3.152(3)$ & $143(3)$ \\
$\mathrm{N} 6-\mathrm{H} 6 A \cdots \mathrm{N} 17^{\mathrm{ii}}$ & $0.85(4)$ & $2.17(3)$ & $2.927(3)$ & $149(3)$ \\
$\mathrm{N} 6-\mathrm{H} 6 B \cdots \mathrm{N} 10^{\text {iii }}$ & $0.85(4)$ & $2.16(4)$ & $2.953(3)$ & $156(3)$ \\
$\mathrm{C} 4-\mathrm{H} 4 B \cdots \mathrm{N} 17^{\text {iv }}$ & 0.99 & 2.59 & $3.440(4)$ & 144 \\
$\mathrm{C} 2-\mathrm{H} 2 A \cdots C g 3^{\text {iv }}$ & 0.99 & 2.87 & $3.653(3)$ & 136 \\
\hline
\end{tabular}

Symmetry codes: (i) $x+\frac{1}{2},-y+\frac{3}{2}, z+\frac{1}{2}$; (ii) $x-\frac{1}{2}, y-\frac{1}{2}, z$; (iii) $x,-y+1, z+\frac{1}{2}$; (iv) $x-\frac{1}{2},-y+\frac{3}{2}, z+\frac{1}{2}$.

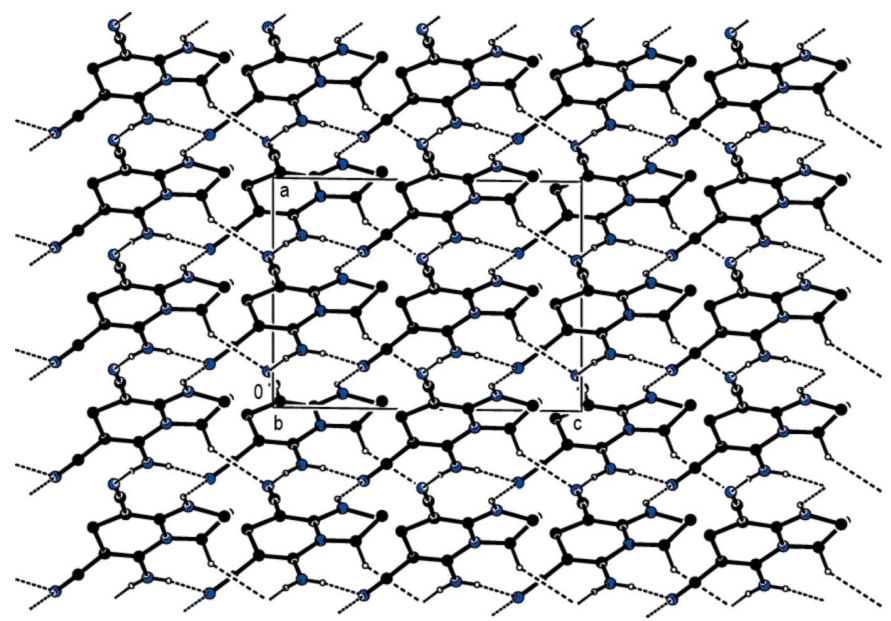

Figure 4

A view along [010] showing the intermolecular $\mathrm{N}-\mathrm{H} \cdots \mathrm{N}$ and $\mathrm{C}-\mathrm{H} \cdots \mathrm{N}$ hydrogen bonds of the title compound. The dichlorophenyl group and $\mathrm{H}$ atoms not involved in hydrogen bonding have been omitted for clarity.

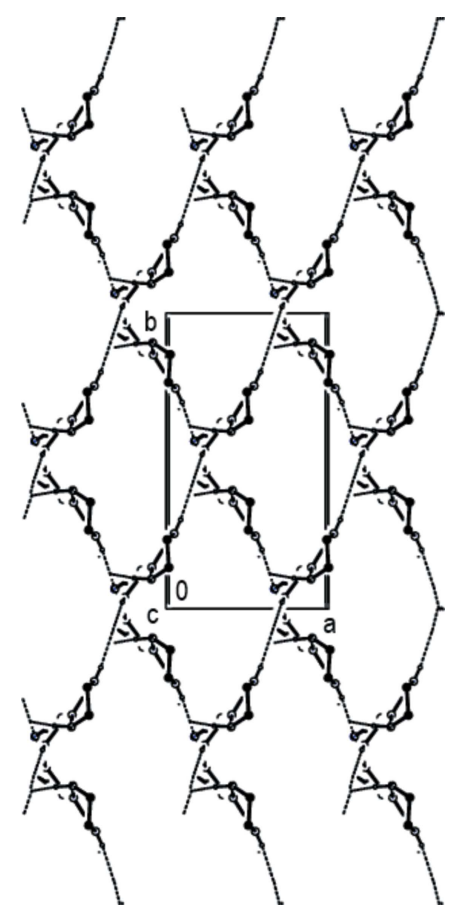

Figure 5

A view along [001] showing the intermolecular $\mathrm{N}-\mathrm{H} \cdots \mathrm{N}$ and $\mathrm{C}-\mathrm{H} \cdots \mathrm{N}$ hydrogen bonds of the title compound. The dichlorophenyl group and $\mathrm{H}$ atoms not involved in hydrogen bonding have been omitted for clarity.
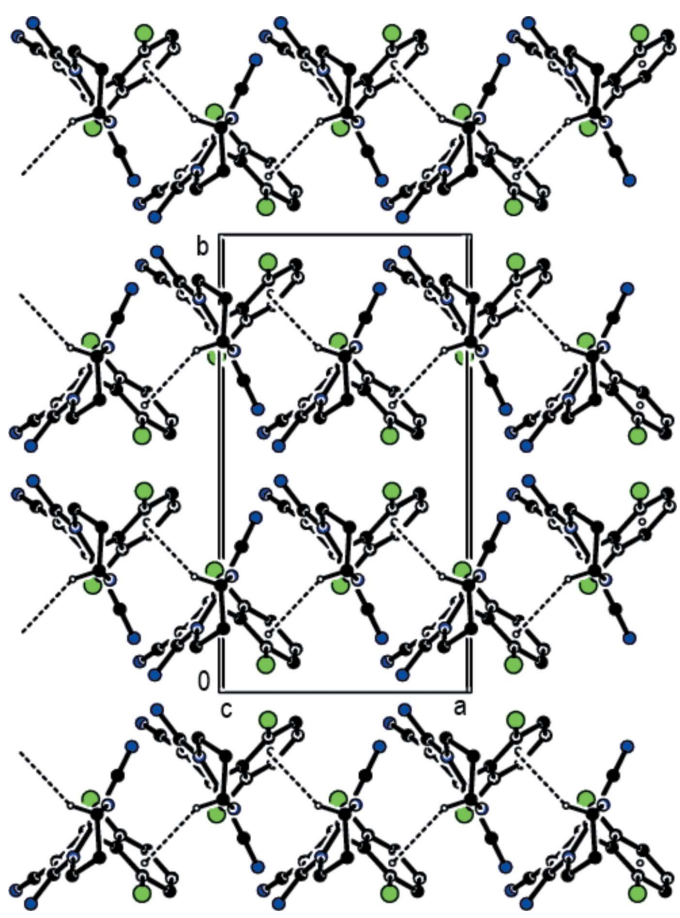

Figure 6

A view along [010] showing the $\mathrm{C}-\mathrm{H} \cdots \pi$ interactions in the title compound.

interactions (Table 1), which form zigzag chains propagating parallel to [100] (Fig. 6), are also involved in the packing.

\section{Hirshfeld surface analysis}

In order to visualize the intermolecular interactions in the crystal of the title compound, a Hirshfeld surface analysis (Spackman \& Jayatilaka, 2009) was performed with CrystalExplorer17.5 (Turner et al., 2017). Fig. 7(a) and Fig. 7(b) show the front and back sides of the three-dimensional Hirshfeld surface of the title molecule plotted over $d_{\text {norm }}$ in the range -0.4776 to +1.4517 a.u., using a 'high standard' surface resolution colour-mapped over the normalized contact distance. The red, white and blue regions visible on the $d_{\text {norm }}$ surfaces indicate contacts with distances shorter, longer and equal to the van der Waals separations. The red spots highlight the

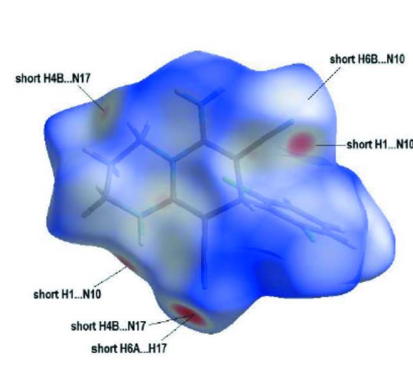

(a)

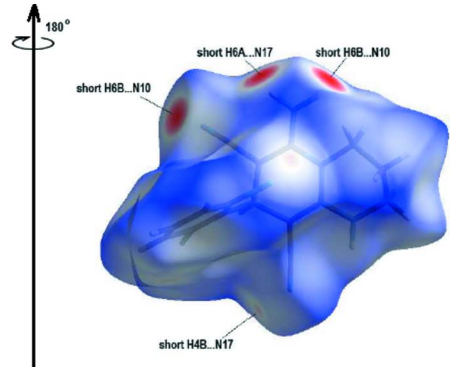

(b)
Figure 7

(a) Front and (b) back sides of the three-dimensional Hirshfeld surface of the title compound plotted over $d_{\text {norm }}$ in the range -0.4776 to +1.4517 a.u. 
Table 2

Summary of short interatomic contacts $(\AA)$ in the title compound.

\begin{tabular}{lll}
\hline Contact & Distance & Symmetry operation \\
\hline $\mathrm{H} 6 B \cdots \mathrm{N} 10$ & 2.16 & $x, 1-y, \frac{1}{2}+z$ \\
$\mathrm{H} 1 \cdots \mathrm{N} 10$ & 2.43 & $\frac{1}{2}+x, \frac{3}{2}-y, \frac{1}{2}+z$ \\
$\mathrm{H} 4 B \cdots \mathrm{N} 17$ & 2.59 & $-\frac{1}{2}+x, \frac{3}{2}-y, \frac{1}{2}+z$ \\
$\mathrm{H} 6 A \cdots \mathrm{N} 17$ & 2.16 & $-\frac{1}{2}+x,-\frac{1}{2}+y, z$ \\
$\mathrm{~N} 10 \cdots \mathrm{H} 15$ & 2.81 & $-1+x, y, z$ \\
$\mathrm{H} 3 B \cdots \mathrm{H} 13$ & 2.57 & $x, y, 1+z$ \\
\hline
\end{tabular}

interatomic contacts, including the $\mathrm{N}-\mathrm{H} \cdots \mathrm{N}$ and $\mathrm{C}-\mathrm{H} \cdots \mathrm{N}$ hydrogen bonds.

The overall two-dimensional fingerprint plot for the title compound and those delineated into $\mathrm{N} \cdots \mathrm{H} / \mathrm{H} \cdots \mathrm{N}, \mathrm{H} \cdots \mathrm{H}$, $\mathrm{C} \cdots \mathrm{H} / \mathrm{H} \cdots \mathrm{C}$ and $\mathrm{Cl} \cdots \mathrm{H} / \mathrm{H} \cdots \mathrm{Cl}$ contacts are illustrated in

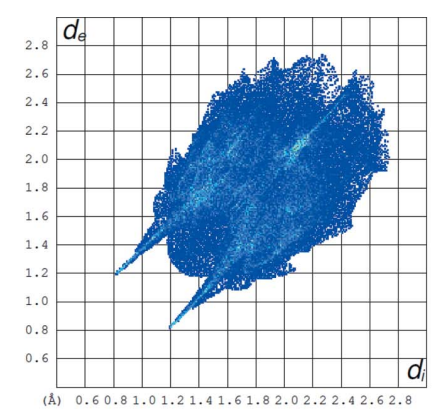

(a) All...All

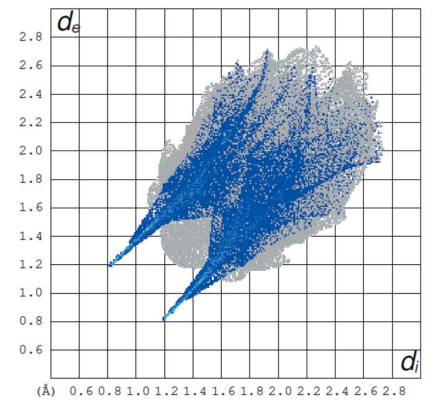

(b) $\mathrm{N} \cdots H / H \cdots N$

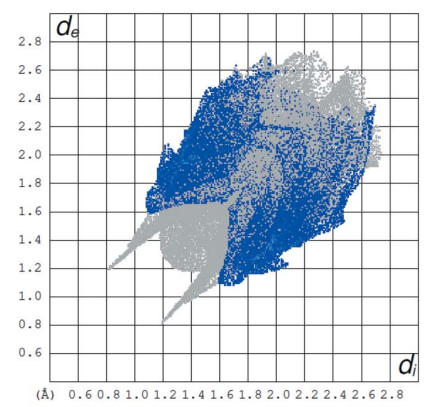

(d) $\mathrm{C} \cdot \boldsymbol{\cdots} / \mathrm{H} \cdot \boldsymbol{\cdots} \mathrm{C}$

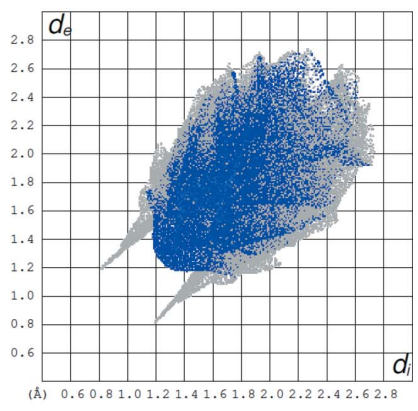

(c) $H$...H

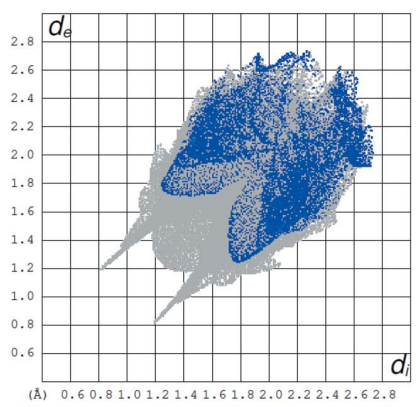

(e) $\mathrm{Cl} \cdots \mathrm{H} / \mathrm{H} \cdots \mathrm{Cl}$
Figure 8

The two-dimensional fingerprint plots of the title compound, showing (a) all interactions, and delineated into $(b) \mathrm{N} \cdots \mathrm{H} / \mathrm{H} \cdots \mathrm{N},(c) \mathrm{H} \cdots \mathrm{H},(d)$ $\mathrm{C} \cdots \mathrm{H} / \mathrm{H} \cdots \mathrm{C}$ and $(f) \mathrm{Cl} \cdots \mathrm{H} / \mathrm{H} \cdots \mathrm{Cl}$ interactions $\left[d_{\mathrm{e}}\right.$ and $d_{\mathrm{i}}$ represent the distances from a point on the Hirshfeld surface to the nearest atoms outside (external) and inside (internal) the surface, respectively].
Table 3

Percentage contributions of interatomic contacts to the Hirshfeld surface for the title compound.

\begin{tabular}{ll}
\hline Contact & Percentage contribution \\
\hline $\mathrm{N} \cdots \mathrm{H} / \mathrm{H} \cdots \mathrm{N}$ & 28.4 \\
$\mathrm{H} \cdots \mathrm{H}$ & 24.5 \\
$\mathrm{C} \cdots \mathrm{H} / \mathrm{H} \cdots \mathrm{C}$ & 21.4 \\
$\mathrm{Cl} \cdots \mathrm{H} / \mathrm{H} \cdots \mathrm{Cl}$ & 16.1 \\
$\mathrm{Cl} \cdots \mathrm{C} / \mathrm{C} \cdots \mathrm{Cl}$ & 3.3 \\
$\mathrm{Cl} \cdots \mathrm{Cl}$ & 2.5 \\
$\mathrm{Cl} \cdots \mathrm{N} / \mathrm{N} \cdots \mathrm{Cl}$ & 2.3 \\
$\mathrm{C} \cdots \mathrm{N} / \mathrm{N} \cdots \mathrm{C}$ & 0.8 \\
$\mathrm{C} \cdots \mathrm{C}$ & 0.6 \\
$\mathrm{~N} \cdots \mathrm{N}$ & 0.2 \\
\hline
\end{tabular}

Fig. 8. Numerical details of the various contacts are given in Table 2 and their percentage contributions to the Hirshfeld surfaces are collated in Table 3. N $\cdots \mathrm{H} / \mathrm{H} \cdots \mathrm{N}(28.4 \%), \mathrm{H} \cdots \mathrm{H}$ $(24.5 \%), \mathrm{C} \cdots \mathrm{H} / \mathrm{H} \cdots \mathrm{C}(21.4 \%)$ and $\mathrm{Cl} \cdots \mathrm{H} / \mathrm{H} \cdots \mathrm{Cl}(16.1 \%)$ contribute significantly to the packing while $\mathrm{Cl} \cdots \mathrm{C} / \mathrm{C} \cdots \mathrm{Cl}$, $\mathrm{Cl} \cdots \mathrm{Cl}, \mathrm{Cl} \cdots \mathrm{N} / \mathrm{N} \cdots \mathrm{Cl}, \mathrm{C} \cdot \mathrm{N} / \mathrm{N} \cdots \mathrm{C}, \quad \mathrm{C} \cdot \mathrm{C}$ and $\mathrm{N} \cdots \mathrm{N}$ contacts have a negligible directional impact.

The large number of $\mathrm{N} \cdots \mathrm{H} / \mathrm{H} \cdots \mathrm{N}, \mathrm{H} \cdots \mathrm{H}, \mathrm{C} \cdots \mathrm{H} / \mathrm{H} \cdots \mathrm{C}$ and $\mathrm{Cl} \cdots \mathrm{H} / \mathrm{H} \cdots \mathrm{Cl}$ interactions suggest that van der Waals interactions and hydrogen bonding play the major roles in the crystal packing (Hathwar et al., 2015).

\section{Database survey}

Four related compounds, which have the 1,3,4,8-tetrahydro$2 \mathrm{H}$-pyrido[1,2-a]pyrimidine ring system of the title compound, were found in a search of the Cambridge Structural Database (CSD version 5.42, update of November 2020; Groom et al., 2016): 9-(4-nitrobenzylidene)-8,9-dihydropyrido[2,3- $d$ ]pyrrolo[1,2-a]pyrimidin-5(7H)-one (refcode VAMBET; Khodjaniyazov \& Ashurov, 2016), 11-(aminomethylidene)-8,9,10,11tetrahydropyrido $\left[2^{\prime}, 3^{\prime}: 4,5\right]$ pyrimido[1,2-a]azepin-5(7H)-one (HECLUZ; Khodjaniyazov et al., 2017), 7'-amino- $1^{\prime} H$-spiro[cycloheptane-1,2'-pyrimido[4,5-d]pyrimidin]-4' $\left(3^{\prime} H\right)$-one (LEGLIU; Chen et al., 2012) and 11-(2-oxopyrrolidin-1ylmethyl)-1,2,3,4,5,6,11,11a-octahydropyrido[2,1- $b$ ] quinazolin-6-one dihydrate (KUTPEV; Samarov et al., 2010).

In the crystal of VAMBET, molecules are linked via $\mathrm{C}-$ $\mathrm{H} \cdots \mathrm{O}$ and $\mathrm{C}-\mathrm{H} \cdots \mathrm{N}$ hydrogen bonds, forming layers parallel to (101). In the molecule of HECLUZ, the seven-membered pentamethylene ring adopts a twist-boat conformation. In the crystal, hydrogen bonds with 16-membered ring and three chain motifs are generated by $\mathrm{N}-\mathrm{H} \cdots \mathrm{N}$ and $\mathrm{N}-\mathrm{H} \cdots \mathrm{O}$ contacts. The amino group is located close to the nitrogen atoms, forming hydrogen bonds with $R_{2}^{1}(4)$ and $R_{2}^{2}(12)$ graphset motifs. This amino group also forms a hydrogen bond with the $\mathrm{C}=\mathrm{O}$ oxygen atom of a molecule translated parallel to [100], which links the molecules into $R_{4}^{4}(16)$ rings. Hydrogenbonded chains are formed along [100] by alternating $R_{2}^{2}(12)$ and $R_{4}^{4}(16)$ rings. These chains are stabilized by intermolecular $\pi-\pi$ stacking interactions observed between the pyridine and pyrimidine rings. In LEGLIU, the molecular structure is built up from two fused six-membered rings and one seven- 
Table 4

Experimental details.

\begin{tabular}{|c|c|}
\hline \multicolumn{2}{|l|}{ Crystal data } \\
\hline Chemical formula & $\mathrm{C}_{16} \mathrm{H}_{13} \mathrm{Cl}_{2} \mathrm{~N}_{5}$ \\
\hline$M_{\mathrm{r}}$ & 346.21 \\
\hline Crystal system, space group & Monoclinic, $C c$ \\
\hline Temperature $(\mathrm{K})$ & 100 \\
\hline$a, b, c(\AA)$ & $8.6598(2), 16.0275(5), 11.6590(3)$ \\
\hline$\beta\left(^{\circ}\right)$ & $90.7364(9)$ \\
\hline$V\left(\AA^{3}\right)$ & $1618.08(8)$ \\
\hline$Z$ & 4 \\
\hline Radiation type & Мо $K \alpha$ \\
\hline$\mu\left(\mathrm{mm}^{-1}\right)$ & 0.41 \\
\hline Crystal size $(\mathrm{mm})$ & $0.30 \times 0.03 \times 0.03$ \\
\hline \multicolumn{2}{|l|}{ Data collection } \\
\hline Diffractometer & $\begin{array}{l}\text { Bruker D8 QUEST PHOTON-III } \\
\text { CCD }\end{array}$ \\
\hline Absorption correction & $\begin{array}{l}\text { Multi-scan (SADABS; Krause et } \\
\quad \text { al., 2015) }\end{array}$ \\
\hline$T_{\min }, T_{\max }$ & $0.880,0.980$ \\
\hline $\begin{array}{l}\text { No. of measured, independent and } \\
\text { observed }[I>2 \sigma(I)] \text { reflections }\end{array}$ & $21346,5861,4528$ \\
\hline$R_{\text {int }}$ & 0.064 \\
\hline$(\sin \theta / \lambda)_{\max }\left(\AA^{-1}\right)$ & 0.758 \\
\hline \multicolumn{2}{|l|}{ Refinement } \\
\hline$R\left[F^{2}>2 \sigma\left(F^{2}\right)\right], w R\left(F^{2}\right), S$ & $0.044,0.090,1.03$ \\
\hline No. of reflections & 5861 \\
\hline No. of parameters & 217 \\
\hline No. of restraints & 2 \\
\hline $\mathrm{H}$-atom treatment & $\begin{array}{l}\mathrm{H} \text { atoms treated by a mixture of } \\
\text { independent and constrained } \\
\text { refinement }\end{array}$ \\
\hline$\Delta \rho_{\max }, \Delta \rho_{\min }\left(\mathrm{e} \AA^{-3}\right)$ & $0.25,-0.32$ \\
\hline Absolute structure & $\begin{array}{l}\text { Flack } x \text { determined using } 1774 \\
\quad \text { quotients }\left[\left(I^{+}\right)-\left(I^{-}\right)\right] /\left[\left(I^{+}\right)+\left(I^{-}\right)\right] \\
\text {(Parsons } \text { et al., 2013). }\end{array}$ \\
\hline Absolute structure parameter & $0.27(3)$ \\
\hline
\end{tabular}

Computer programs: APEX3 (Bruker, 2018), SAINT (Bruker, 2013), SHELXT2014/5 (Sheldrick, 2015a), SHELXL2018/3 (Sheldrick, 2015b), ORTEP-3 for Windows (Farrugia, 2012) and PLATON (Spek, 2020).

membered ring linked through a spiro $\mathrm{C}$ atom. The crystal packing is stabilized by intermolecular $\mathrm{N}-\mathrm{H} \cdots \mathrm{O}$ hydrogen bonds between the two $\mathrm{N}-\mathrm{H}$ groups and the ketone $\mathrm{O}$ atoms of the neighbouring molecules. In KUTPEV, water molecules are mutually $\mathrm{O}-\mathrm{H} \cdots \mathrm{O}$ hydrogen bonded and form infinite chains propagating parallel to [010]. Neighbouring chains are linked by the quinazoline molecules by means of $\mathrm{O}-$ $\mathrm{H} \cdots \mathrm{O}=\mathrm{C}$ hydrogen bonds, forming a two-dimensional network.

\section{Synthesis and crystallization}

To a dissolved mixture of 2-(2,6-dichlorobenzylidene)malononitrile $(1.14 \mathrm{~g} ; 5.1 \mathrm{mmol})$ and malononitrile $(0.34 \mathrm{~g} ; 5.2 \mathrm{mmol})$ in methanol $(40 \mathrm{~mL}), 1,3$-diaminopropane $(0.38 \mathrm{~g} ; 5.2 \mathrm{mmol})$ was added and was stirred at room temperature for $10 \mathrm{~min}$. Then $25 \mathrm{~mL}$ of methanol were removed from the reaction mixture that was left overnight. The precipitated crystals were separated by filtration and recrystallized from ethanol (yield 78\%; m.p. 541-542 K).

${ }^{1} \mathrm{H}$ NMR (300 MHz, DMSO- $\left.d_{6}\right): 1.89\left(m, 2 \mathrm{H}, \mathrm{CH}_{2}\right) ; 3.13(m$, $\left.2 \mathrm{H}, \mathrm{CH}_{2}\right) ; 3.67\left(m, 2 \mathrm{H}, \mathrm{CH}_{2}\right) ; 5.31$ ( $\left.s, 1 \mathrm{H}, \mathrm{CH}-\mathrm{Ar}\right) ; 6.14(s, 2 \mathrm{H}$,
$\left.\mathrm{NH}_{2}\right) ; 6.78(s, 1 \mathrm{H}, \mathrm{NH}) ; 7.25\left(t, 1 \mathrm{H}, \mathrm{Ar}-\mathrm{H},{ }^{3} J_{\mathrm{H}-\mathrm{H}}=7,9\right) ; 7.42(d$, $\left.2 \mathrm{H}, 2 \mathrm{Ar}-\mathrm{H},{ }^{3} J_{\mathrm{H}-\mathrm{H}}=7,8\right) .{ }^{13} \mathrm{C} \mathrm{NMR}\left(75 \mathrm{MHz}, \mathrm{DMSO}-d_{6}\right): 22.30$ $\left(\mathrm{CH}_{2}\right), 36.32(\mathrm{Ar}-\mathrm{CH}), 38.62\left(\mathrm{CH}_{2}\right), 42.92\left(\mathrm{CH}_{2}\right), 51.70$ (= $\left.\mathrm{C}_{\text {quar }}\right), 55.06\left(=\mathrm{C}_{\text {quar }}\right), 121.61(\mathrm{CN}), 122.04(\mathrm{CN}), 129.56$ $\left(3 \mathrm{CH}_{\text {arom }}\right), 138.25\left(3 \mathrm{C}_{\mathrm{ar}}\right), 152.11\left(=\mathrm{C}_{\text {quar }}\right), 154.17\left(=\mathrm{C}_{\text {quar }}\right)$.

\section{Refinement}

Crystal data, data collection and structure refinement details are summarized in Table 4 . The $\mathrm{C}$-bound $\mathrm{H}$ atoms were placed in calculated positions $(\mathrm{C}-\mathrm{H}=0.95-1.00 \AA)$ and refined as riding with $U_{\text {iso }}(\mathrm{H})=1.2 U_{\text {eq }}(\mathrm{C})$. All $\mathrm{N}$-bound $\mathrm{H}$ atoms were located in a difference map $[\mathrm{N} 1-\mathrm{H} 1=0.85$ (3) $\AA$, N6-H6 $A=$ 0.85 (4) $\AA$ and $\mathrm{N} 6-\mathrm{H} 6 B=0.85$ (4) $\AA$ ] and they were refined with the constraint $U_{\text {iso }}(\mathrm{H})=1.2 U_{\text {eq }}(\mathrm{N})$.

\section{Acknowledgements}

Authors contributions are as follows. Conceptualization, FNN and IGM; methodology, FNN and IGM; investigation, VNK, FNN, TAT and AAA; writing (original draft), MA and IGM; writing (review and editing of the manuscript), MA and IGM; visualization, MA, FNN and IGM; funding acquisition, VNK and FNN; resources, RMR, AAA and FNN; supervision, IGM and MA.

\section{Funding information}

This work was supported by Baku State University, and RUDN University Strategic Academic Leadership Program.

\section{References}

Abdelhamid, A. A., Mohamed, S. K., Khalilov, A. N., Gurbanov, A. V. \& Ng, S. W. (2011). Acta Cryst. E67, o744.

Abdelhamid, A. A., Mohamed, S. K., Maharramov, A. M., Khalilov, A. N. \& Allahverdiev, M. A. (2014). J. Saudi Chem. Soc. 18, 474478.

Awouters, F., Vermeire, J., Smeyers, F., Vermote, P., van Beek, R. \& Niemegeers, C. J. E. (1986). Drug Dev. Res. 8, 95-102.

Blaton, N. M., Peeters, O. M. \& De Ranter, C. J. (1995). Acta Cryst. C51, 533-535.

Bruker (2013). SAINT. Bruker AXS Inc., Madison, Wisconsin, USA.

Bruker (2018). APEX3. Bruker AXS Inc., Madison, Wisconsin, USA.

Chen, S., Shi, D., Liu, M. \& Li, J. (2012). Acta Cryst. E68, o2546.

Cremer, D. \& Pople, J. A. (1975). J. Am. Chem. Soc. 97, 1354-1358.

Farrugia, L. J. (2012). J. Appl. Cryst. 45, 849-854.

Groom, C. R., Bruno, I. J., Lightfoot, M. P. \& Ward, S. C. (2016). Acta Cryst. B72, 171-179.

Hathwar, V. R., Sist, M., Jørgensen, M. R. V., Mamakhel, A. H., Wang, X., Hoffmann, C. M., Sugimoto, K., Overgaard, J. \& Iversen, B. B. (2015). IUCrJ, 2, 563-574.

Hermecz, I. \& Mészáros, Z. (1988). Med. Res. Rev. 8, 203-230.

Janssen, G. V., van den Heuvel, J. A. C., Megens, R. P., Benningshof, J. C. J. \& Ovaa, H. (2018). Bioorg. Med. Chem. 26, 41-49.

Khalilov, A. N., Abdelhamid, A. A., Gurbanov, A. V. \& Ng, S. W. (2011). Acta Cryst. E67, o1146.

Khodjaniyazov, Kh. U. \& Ashurov, J. M. (2016). Acta Cryst. E72, 452455.

Khodjaniyazov, K. U., Makhmudov, U. S., Turgunov, K. K. \& Elmuradov, B. Z. (2017). Acta Cryst. E73, 1497-1500. 
Krause, L., Herbst-Irmer, R., Sheldrick, G. M. \& Stalke, D. (2015). J. Appl. Cryst. 48, 3-10.

Mahmoudi, G., Dey, L., Chowdhury, H., Bauzá, A., Ghosh, B. K., Kirillov, A. M., Seth, S. K., Gurbanov, A. V. \& Frontera, A. (2017). Inorg. Chim. Acta, 461, 192-205.

Mahmoudi, G., Khandar, A. A., Afkhami, F. A., Miroslaw, B., Gurbanov, A. V., Zubkov, F. I., Kennedy, A., Franconetti, A. \& Frontera, A. (2019). CrystEngComm, 21, 108-117.

Mamedov, I. G., Bayramov, M. R., Mamedova, Y. V. \& Maharramov, A. M. (2013). Magn. Reson. Chem. 51, 234-239.

McLaughlin, E. C., Norman, M. W., Ko Ko, T. \& Stolt, I. (2014). Tetrahedron Lett. 55, 2609-2611.

Naghiyev, F. N., Akkurt, M., Askerov, R. K., Mamedov, I. G., Rzayev, R. M., Chyrka, T. \& Maharramov, A. M. (2020a). Acta Cryst. E76, 720-723.

Naghiyev, F. N., Cisterna, J., Khalilov, A. N., Maharramov, A. M., Askerov, R. K., Asadov, K. A., Mamedov, I. G., Salmanli, K. S., Cárdenas, A. \& Brito, I. (2020b). Molecules, 25, 2235.

Naghiyev, F. N., Grishina, M. M., Khrustalev, V. N., Khalilov, A. N., Akkurt, M., Akobirshoeva, A. A. \& Mamedov, İ. G. (2021). Acta Cryst. E77, 195-199.

Naghiyev, F. N., Mammadova, G. Z., Mamedov, I. G., Huseynova, A. T., Çelikesir, S. T., Akkurt, M. \& Akobirshoeva, A. A. (2020c). Acta Cryst. E76, 1365-1368.

Parsons, S., Flack, H. D. \& Wagner, T. (2013). Acta Cryst. B69, 249259.

Pryadeina, M. V., Burgart, Y. V., Kodess, M. I. \& Saloutin, V. I. (2005). Russ. Chem. Bull. 54, 2841-2845.
Riva, R., Banfi, L., Castaldi, G., Ghislieri, D., Malpezzi, L., Musumeci, F., Tufaro, R. \& Rasparini, M. (2011). Eur. J. Org. Chem. pp. 23192325.

Samarov, Z. U., Okmanov, R. Y., Turgunov, K. K., Tashkhodjaev, B. \& Shakhidoyatov, K. M. (2010). Acta Cryst. E66, o890.

Sheldrick, G. M. (2015a). Acta Cryst. A71, 3-8.

Sheldrick, G. M. (2015b). Acta Cryst. C71, 3-8.

Spackman, M. A. \& Jayatilaka, D. (2009). CrystEngComm, 11, 1932.

Spek, A. L. (2020). Acta Cryst. E76, 1-11.

Turner, M. J., Mckinnon, J. J., Wolff, S. K., Grimwood, D. J., Spackman, P. R., Jayatilaka, D. \& Spackman, M. A. (2017). CrystalExplorer17. University of Western Australia.

Ukrainets, I. V., Bereznyakova, N. L., Sim, G. \& Davidenko, A. A. (2018). Pharm. Chem. J. 52, 601-605.

Viswanathan, A., Kute, D., Musa, A., Mani, S. K., Sipilä, V., EmmertStreib, F., Zubkov, F. I., Gurbanov, A. V., Yli-Harja, O. \& Kandhavelu, M. (2019). Eur. J. Med. Chem. 166, 291-303.

Wu, Y.-J., He, H., Hu, S., Huang, Y., Scola, P. M., Grant-Young, K., Bertekap, R. L., Wu, D., Gao, Q., Li, Y., Klakouski, C. \& Westphal, R. S. (2003). J. Med. Chem. 46, 4834-4837.

Yadigarov, R. R., Khalilov, A. N., Mamedov, I. G., Nagiev, F. N., Magerramov, A. M. \& Allakhverdiev, M. A. (2009). Russ. J. Org. Chem. 45, 1856-1858.

Yahata, H., Saito, M., Sendo, T., Itoh, Y., Uchida, M., Hirakawa, T., Nakano, H. \& Oishi, R. (2006). Int. J. Cancer, 118, 2636-2638.

Yin, J., Khalilov, A. N., Muthupandi, P., Ladd, R. \& Birman, V. B. (2020). J. Am. Chem. Soc. 142, 60-63. 


\section{supporting information}

Acta Cryst. (2021). E77, 516-521 [https://doi.org/10.1107/S2056989021003583]

Crystal structure and Hirshfeld surface analysis of 6-amino-8-(2,6-dichlorophenyl)-1,3,4,8-tetrahydro-2H-pyrido[1,2-a]pyrimidine-7,9-dicarbonitrile

Farid N. Naghiyev, Tatiana A. Tereshina, Victor N. Khrustalev, Mehmet Akkurt, Rovnag M.

Rzayev, Anzurat A. Akobirshoeva and İbrahim G. Mamedov

Computing details

Data collection: APEX3 (Bruker, 2018); cell refinement: SAINT (Bruker, 2013); data reduction: SAINT (Bruker, 2013); program(s) used to solve structure: SHELXT2014/5 (Sheldrick, 2015a); program(s) used to refine structure:

SHELXL2018/3 (Sheldrick, 2015b); molecular graphics: ORTEP-3 for Windows (Farrugia, 2012); software used to prepare material for publication: PLATON (Spek, 2020).

6-Amino-8-(2,6-dichlorophenyl)-1,3,4,8-tetrahydro-2H-pyrido[1,2-a]pyrimidine-7,9-dicarbonitrile

Crystal data

$\mathrm{C}_{16} \mathrm{H}_{13} \mathrm{Cl}_{2} \mathrm{~N}_{5}$

$M_{r}=346.21$

Monoclinic, $C c$

$a=8.6598(2) \AA$

$b=16.0275(5) \AA$

$c=11.6590(3) \AA$

$\beta=90.7364(9)^{\circ}$

$V=1618.08(8) \AA^{3}$

$Z=4$

\section{Data collection}

Bruker D8 QUEST PHOTON-III CCD diffractometer

$\varphi$ and $\omega$ scans

Absorption correction: multi-scan

(SADABS; Krause et al., 2015)

$T_{\min }=0.880, T_{\max }=0.980$

21346 measured reflections

\section{Refinement}

Refinement on $F^{2}$

Least-squares matrix: full

$R\left[F^{2}>2 \sigma\left(F^{2}\right)\right]=0.044$

$w R\left(F^{2}\right)=0.090$

$S=1.02$

5861 reflections

217 parameters

2 restraints
$F(000)=712$

$D_{\mathrm{x}}=1.421 \mathrm{Mg} \mathrm{m}^{-3}$

Mo $K \alpha$ radiation, $\lambda=0.71073 \AA$

Cell parameters from 4611 reflections

$\theta=2.5-32.2^{\circ}$

$\mu=0.41 \mathrm{~mm}^{-1}$

$T=100 \mathrm{~K}$

Needle, colourless

$0.30 \times 0.03 \times 0.03 \mathrm{~mm}$

5861 independent reflections

4528 reflections with $I>2 \sigma(I)$

$R_{\text {int }}=0.064$

$\theta_{\text {max }}=32.6^{\circ}, \theta_{\min }=2.5^{\circ}$

$h=-13 \rightarrow 13$

$k=-24 \rightarrow 24$

$l=-17 \rightarrow 17$

Primary atom site location: difference Fourier map

Secondary atom site location: difference Fourier map

Hydrogen site location: mixed

$\mathrm{H}$ atoms treated by a mixture of independent and constrained refinement

$w=1 /\left[\sigma^{2}\left(F_{\mathrm{o}}^{2}\right)+(0.0315 P)^{2}+0.2854 P\right]$

where $P=\left(F_{\mathrm{o}}^{2}+2 F_{\mathrm{c}}^{2}\right) / 3$ 
$(\Delta / \sigma)_{\max }<0.001$

$\Delta \rho_{\max }=0.25 \mathrm{e} \AA^{-3}$

$\Delta \rho_{\min }=-0.32$ e $\AA^{-3}$
Absolute structure: Flack $x$ determined using

1774 quotients $\left[\left(I^{+}\right)-\left(I^{\prime}\right)\right] /\left[\left(I^{+}\right)+\left(I^{-}\right)\right]$(Parsons et al., 2013).

Absolute structure parameter: 0.27 (3)

\section{Special details}

Geometry. All esds (except the esd in the dihedral angle between two 1.s. planes) are estimated using the full covariance matrix. The cell esds are taken into account individually in the estimation of esds in distances, angles and torsion angles; correlations between esds in cell parameters are only used when they are defined by crystal symmetry. An approximate (isotropic) treatment of cell esds is used for estimating esds involving l.s. planes.

Fractional atomic coordinates and isotropic or equivalent isotropic displacement parameters $\left(\AA^{2}\right)$

\begin{tabular}{|c|c|c|c|c|}
\hline & $x$ & $y$ & $z$ & $U_{\text {iso }} * / U_{\text {eq }}$ \\
\hline $\mathrm{Cl1}$ & $0.48473(9)$ & $0.76604(6)$ & $0.16871(7)$ & $0.0429(2)$ \\
\hline $\mathrm{Cl} 2$ & $0.69787(9)$ & $0.56058(4)$ & $0.51124(7)$ & $0.03080(17)$ \\
\hline N1 & $0.5635(3)$ & $0.75406(14)$ & $0.7250(2)$ & $0.0199(5)$ \\
\hline $\mathrm{H} 1$ & $0.604(4)$ & $0.801(2)$ & 0.709 (3) & $0.024^{*}$ \\
\hline $\mathrm{C} 2$ & $0.5218(3)$ & $0.73373(16)$ & $0.8418(2)$ & $0.0207(5)$ \\
\hline $\mathrm{H} 2 \mathrm{~A}$ & 0.4154 & 0.7528 & 0.8576 & $0.025^{*}$ \\
\hline $\mathrm{H} 2 \mathrm{~B}$ & 0.5934 & 0.7611 & 0.8969 & $0.025^{*}$ \\
\hline $\mathrm{C} 3$ & $0.5326(4)$ & 0.63931 (17) & $0.8531(2)$ & $0.0248(6)$ \\
\hline $\mathrm{H} 3 \mathrm{~A}$ & 0.6392 & 0.6206 & 0.8373 & $0.030^{*}$ \\
\hline H3B & 0.5064 & 0.6222 & 0.9321 & $0.030^{*}$ \\
\hline C4 & 0.4209 (3) & $0.59988(16)$ & $0.7681(2)$ & $0.0218(5)$ \\
\hline $\mathrm{H} 4 \mathrm{~A}$ & 0.4471 & 0.5401 & 0.7595 & $0.026^{*}$ \\
\hline $\mathrm{H} 4 \mathrm{~B}$ & 0.3151 & 0.6033 & 0.7990 & $0.026^{*}$ \\
\hline N5 & $0.4232(3)$ & 0.64027 (13) & $0.65394(19)$ & 0.0167 (4) \\
\hline C6 & $0.3418(3)$ & $0.60234(14)$ & $0.5649(2)$ & $0.0163(5)$ \\
\hline N6 & $0.2511(3)$ & $0.53821(15)$ & $0.5930(2)$ & $0.0236(5)$ \\
\hline H6A & $0.214(4)$ & $0.506(2)$ & $0.541(3)$ & $0.028^{*}$ \\
\hline H6B & $0.238(4)$ & $0.522(2)$ & $0.661(3)$ & $0.028^{*}$ \\
\hline $\mathrm{C} 7$ & $0.3535(3)$ & $0.63028(15)$ & $0.4540(2)$ & $0.0155(5)$ \\
\hline $\mathrm{C} 8$ & 0.4667 (3) & $0.69614(15)$ & $0.4164(2)$ & $0.0167(5)$ \\
\hline H8 & 0.4066 & 0.7379 & 0.3704 & $0.020^{*}$ \\
\hline C9 & $0.5266(3)$ & $0.74104(16)$ & $0.5222(2)$ & $0.0179(5)$ \\
\hline C9A & $0.5062(3)$ & $0.71289(15)$ & $0.6322(2)$ & $0.0161(5)$ \\
\hline $\mathrm{C} 10$ & $0.2600(3)$ & $0.59393(15)$ & $0.3682(2)$ & $0.0147(5)$ \\
\hline N10 & $0.1851(3)$ & $0.56730(13)$ & $0.2941(2)$ & $0.0194(5)$ \\
\hline C11 & $0.5914(3)$ & 0.66149 (18) & $0.3380(3)$ & $0.0200(5)$ \\
\hline $\mathrm{C} 12$ & $0.6051(3)$ & $0.6880(2)$ & $0.2246(3)$ & $0.0283(6)$ \\
\hline $\mathrm{C} 13$ & $0.7126(4)$ & 0.6549 (3) & $0.1492(3)$ & $0.0375(8)$ \\
\hline H13 & 0.7167 & 0.6742 & 0.0723 & $0.045^{*}$ \\
\hline C14 & $0.8127(4)$ & $0.5940(2)$ & $0.1873(3)$ & $0.0383(8)$ \\
\hline H14 & 0.8869 & 0.5715 & 0.1367 & $0.046^{*}$ \\
\hline $\mathrm{C} 15$ & 0.8058 (3) & 0.56565 (19) & $0.2989(3)$ & $0.0314(7)$ \\
\hline H15 & 0.8746 & 0.5235 & 0.3254 & $0.038^{*}$ \\
\hline C16 & $0.6971(3)$ & $0.59938(18)$ & 0.3718 (3) & $0.0240(6)$ \\
\hline $\mathrm{C} 17$ & 0.5995 (3) & $0.81835(17)$ & $0.5040(2)$ & $0.0228(6)$ \\
\hline
\end{tabular}


Atomic displacement parameters $\left(\AA^{2}\right)$

\begin{tabular}{lllllll}
\hline & $U^{11}$ & $U^{22}$ & $U^{33}$ & $U^{12}$ & $U^{13}$ & $U^{23}$ \\
\hline C11 & $0.0391(4)$ & $0.0681(6)$ & $0.0215(4)$ & $-0.0012(4)$ & $0.0009(3)$ & $0.0190(4)$ \\
C12 & $0.0305(4)$ & $0.0267(3)$ & $0.0353(4)$ & $0.0081(3)$ & $0.0050(3)$ & $0.0077(3)$ \\
N1 & $0.0294(12)$ & $0.0147(10)$ & $0.0156(11)$ & $-0.0072(9)$ & $0.0006(9)$ & $0.0002(8)$ \\
C2 & $0.0278(14)$ & $0.0215(13)$ & $0.0127(12)$ & $-0.0055(10)$ & $-0.0012(10)$ & $-0.0013(10)$ \\
C3 & $0.0353(16)$ & $0.0207(13)$ & $0.0180(14)$ & $-0.0050(11)$ & $-0.0071(12)$ & $0.0033(10)$ \\
C4 & $0.0328(14)$ & $0.0189(12)$ & $0.0136(13)$ & $-0.0088(11)$ & $-0.0029(11)$ & $0.0027(9)$ \\
N5 & $0.0237(11)$ & $0.0145(10)$ & $0.0120(10)$ & $-0.0050(8)$ & $0.0000(8)$ & $0.0003(7)$ \\
C6 & $0.0200(12)$ & $0.0127(10)$ & $0.0161(12)$ & $-0.0016(9)$ & $-0.0006(9)$ & $-0.0018(9)$ \\
N6 & $0.0354(13)$ & $0.0211(11)$ & $0.0142(11)$ & $-0.0141(10)$ & $-0.0024(10)$ & $0.0009(9)$ \\
C7 & $0.0179(11)$ & $0.0150(11)$ & $0.0137(12)$ & $-0.0006(9)$ & $0.0007(9)$ & $-0.0013(9)$ \\
C8 & $0.0216(13)$ & $0.0135(10)$ & $0.0151(12)$ & $-0.0022(9)$ & $0.0001(10)$ & $0.0002(9)$ \\
C9 & $0.0242(13)$ & $0.0149(11)$ & $0.0148(13)$ & $-0.0040(9)$ & $0.0029(10)$ & $-0.0025(9)$ \\
C9A & $0.0189(12)$ & $0.0131(10)$ & $0.0163(12)$ & $-0.0025(9)$ & $0.0001(9)$ & $-0.0007(9)$ \\
C10 & $0.0179(11)$ & $0.0123(10)$ & $0.0140(12)$ & $0.0010(9)$ & $0.0034(9)$ & $0.0018(8)$ \\
N10 & $0.0226(11)$ & $0.0193(11)$ & $0.0163(12)$ & $0.0030(9)$ & $-0.0013(9)$ & $-0.0016(8)$ \\
C11 & $0.0220(12)$ & $0.0199(11)$ & $0.0181(12)$ & $-0.0082(9)$ & $0.0022(10)$ & $-0.0051(9)$ \\
C12 & $0.0257(15)$ & $0.0417(17)$ & $0.0175(15)$ & $-0.0130(13)$ & $0.0008(11)$ & $-0.0018(12)$ \\
C13 & $0.0281(16)$ & $0.067(2)$ & $0.0179(15)$ & $-0.0197(16)$ & $0.0048(12)$ & $-0.0117(15)$ \\
C14 & $0.0248(15)$ & $0.053(2)$ & $0.038(2)$ & $-0.0131(15)$ & $0.0123(13)$ & $-0.0255(16)$ \\
C15 & $0.0214(14)$ & $0.0300(15)$ & $0.043(2)$ & $-0.0064(12)$ & $0.0079(13)$ & $-0.0150(14)$ \\
C16 & $0.0228(14)$ & $0.0229(13)$ & $0.0264(15)$ & $-0.0039(10)$ & $0.0037(11)$ & $-0.0039(11)$ \\
C17 & $0.0329(15)$ & $0.0213(12)$ & $0.0144(13)$ & $-0.0073(11)$ & $0.0054(11)$ & $-0.0053(10)$ \\
N17 & $0.064(2)$ & $0.0259(12)$ & $0.0189(13)$ & $-0.0208(13)$ & $0.0093(12)$ & $-0.0046(10)$ \\
& & & & & &
\end{tabular}

Geometric parameters $\left(\AA,{ }^{\circ}\right)$

\begin{tabular}{llll}
\hline $\mathrm{C} 11-\mathrm{C} 12$ & $1.749(4)$ & $\mathrm{N} 6-\mathrm{H} 6 \mathrm{~B}$ & $0.85(4)$ \\
$\mathrm{C} 12-\mathrm{C} 16$ & $1.741(3)$ & $\mathrm{C} 7-\mathrm{C} 10$ & $1.406(4)$ \\
$\mathrm{N} 1-\mathrm{C} 9 \mathrm{~A}$ & $1.355(3)$ & $\mathrm{C} 7-\mathrm{C} 8$ & $1.509(3)$ \\
$\mathrm{N} 1-\mathrm{C} 2$ & $1.451(3)$ & $\mathrm{C} 8-\mathrm{C} 9$ & $1.514(4)$ \\
$\mathrm{N} 1-\mathrm{H} 1$ & $0.85(3)$ & $\mathrm{C} 8-\mathrm{C} 11$ & $1.529(4)$ \\
$\mathrm{C} 2-\mathrm{C} 3$ & $1.522(4)$ & $\mathrm{C} 8-\mathrm{H} 8$ & 1.0000 \\
$\mathrm{C} 2-\mathrm{H} 2 \mathrm{~A}$ & 0.9900 & $\mathrm{C} 9-\mathrm{C} 9 \mathrm{~A}$ & $1.373(4)$ \\
$\mathrm{C} 2-\mathrm{H} 2 \mathrm{~B}$ & 0.9900 & $\mathrm{C} 9-\mathrm{C} 17$ & $1.408(4)$ \\
$\mathrm{C} 3-\mathrm{C} 4$ & $1.514(4)$ & $\mathrm{C} 10-\mathrm{N} 10$ & $1.155(3)$ \\
$\mathrm{C} 3-\mathrm{H} 3 \mathrm{~A}$ & 0.9900 & $\mathrm{C} 11-\mathrm{C} 12$ & $1.395(4)$ \\
$\mathrm{C} 3-\mathrm{H} 3 \mathrm{~B}$ & 0.9900 & $\mathrm{C} 11-\mathrm{C} 16$ & $1.405(4)$ \\
$\mathrm{C} 4-\mathrm{N} 5$ & $1.481(3)$ & $\mathrm{C} 12-\mathrm{C} 13$ & $1.392(4)$ \\
$\mathrm{C} 4-\mathrm{H} 4 \mathrm{~A}$ & 0.9900 & $\mathrm{C} 13-\mathrm{C} 14$ & $1.375(5)$ \\
$\mathrm{C} 4-\mathrm{H} 4 \mathrm{~B}$ & 0.9900 & $\mathrm{C} 13-\mathrm{H} 13$ & 0.9500 \\
$\mathrm{~N} 5-\mathrm{C} 6$ & $1.387(3)$ & $\mathrm{C} 14-\mathrm{C} 15$ & $1.380(5)$ \\
$\mathrm{N} 5-\mathrm{C} 9 \mathrm{~A}$ & $1.393(3)$ & $\mathrm{C} 14-\mathrm{H} 14$ & 0.9500 \\
$\mathrm{C} 6-\mathrm{N} 6$ & $1.337(3)$ & $\mathrm{C} 15-\mathrm{C} 16$ & $1.385(4)$
\end{tabular}




\begin{tabular}{|c|c|c|c|}
\hline $\mathrm{C} 6-\mathrm{C} 7$ & $1.373(4)$ & C15-H15 & 0.9500 \\
\hline N6-H6A & $0.85(4)$ & $\mathrm{C} 17-\mathrm{N} 17$ & $1.150(3)$ \\
\hline $\mathrm{C} 9 \mathrm{~A}-\mathrm{N} 1-\mathrm{C} 2$ & $123.1(2)$ & $\mathrm{C} 7-\mathrm{C} 8-\mathrm{C} 9$ & $108.2(2)$ \\
\hline $\mathrm{C} 9 \mathrm{~A}-\mathrm{N} 1-\mathrm{H} 1$ & $114(2)$ & $\mathrm{C} 7-\mathrm{C} 8-\mathrm{C} 11$ & $112.7(2)$ \\
\hline $\mathrm{C} 2-\mathrm{N} 1-\mathrm{H} 1$ & $121(2)$ & $\mathrm{C} 9-\mathrm{C} 8-\mathrm{C} 11$ & $115.0(2)$ \\
\hline $\mathrm{N} 1-\mathrm{C} 2-\mathrm{C} 3$ & $106.8(2)$ & $\mathrm{C} 7-\mathrm{C} 8-\mathrm{H} 8$ & 106.8 \\
\hline $\mathrm{N} 1-\mathrm{C} 2-\mathrm{H} 2 \mathrm{~A}$ & 110.4 & $\mathrm{C} 9-\mathrm{C} 8-\mathrm{H} 8$ & 106.8 \\
\hline $\mathrm{C} 3-\mathrm{C} 2-\mathrm{H} 2 \mathrm{~A}$ & 110.4 & $\mathrm{C} 11-\mathrm{C} 8-\mathrm{H} 8$ & 106.8 \\
\hline $\mathrm{N} 1-\mathrm{C} 2-\mathrm{H} 2 \mathrm{~B}$ & 110.4 & $\mathrm{C} 9 \mathrm{~A}-\mathrm{C} 9-\mathrm{C} 17$ & $119.5(2)$ \\
\hline $\mathrm{C} 3-\mathrm{C} 2-\mathrm{H} 2 \mathrm{~B}$ & 110.4 & $\mathrm{C} 9 \mathrm{~A}-\mathrm{C} 9-\mathrm{C} 8$ & $123.9(2)$ \\
\hline $\mathrm{H} 2 \mathrm{~A}-\mathrm{C} 2-\mathrm{H} 2 \mathrm{~B}$ & 108.6 & $\mathrm{C} 17-\mathrm{C} 9-\mathrm{C} 8$ & $116.4(2)$ \\
\hline $\mathrm{C} 4-\mathrm{C} 3-\mathrm{C} 2$ & $108.7(2)$ & $\mathrm{N} 1-\mathrm{C} 9 \mathrm{~A}-\mathrm{C} 9$ & $122.4(2)$ \\
\hline $\mathrm{C} 4-\mathrm{C} 3-\mathrm{H} 3 \mathrm{~A}$ & 110.0 & $\mathrm{~N} 1-\mathrm{C} 9 \mathrm{~A}-\mathrm{N} 5$ & $116.5(2)$ \\
\hline $\mathrm{C} 2-\mathrm{C} 3-\mathrm{H} 3 \mathrm{~A}$ & 110.0 & $\mathrm{C} 9-\mathrm{C} 9 \mathrm{~A}-\mathrm{N} 5$ & $121.1(2)$ \\
\hline $\mathrm{C} 4-\mathrm{C} 3-\mathrm{H} 3 \mathrm{~B}$ & 110.0 & $\mathrm{~N} 10-\mathrm{C} 10-\mathrm{C} 7$ & $176.6(3)$ \\
\hline $\mathrm{C} 2-\mathrm{C} 3-\mathrm{H} 3 \mathrm{~B}$ & 110.0 & $\mathrm{C} 12-\mathrm{C} 11-\mathrm{C} 16$ & $114.7(3)$ \\
\hline $\mathrm{H} 3 \mathrm{~A}-\mathrm{C} 3-\mathrm{H} 3 \mathrm{~B}$ & 108.3 & $\mathrm{C} 12-\mathrm{C} 11-\mathrm{C} 8$ & $121.7(3)$ \\
\hline $\mathrm{N} 5-\mathrm{C} 4-\mathrm{C} 3$ & $113.0(2)$ & $\mathrm{C} 16-\mathrm{C} 11-\mathrm{C} 8$ & $123.5(3)$ \\
\hline $\mathrm{N} 5-\mathrm{C} 4-\mathrm{H} 4 \mathrm{~A}$ & 109.0 & $\mathrm{C} 13-\mathrm{C} 12-\mathrm{C} 11$ & $123.2(3)$ \\
\hline $\mathrm{C} 3-\mathrm{C} 4-\mathrm{H} 4 \mathrm{~A}$ & 109.0 & $\mathrm{C} 13-\mathrm{C} 12-\mathrm{Cl}$ & $115.9(3)$ \\
\hline $\mathrm{N} 5-\mathrm{C} 4-\mathrm{H} 4 \mathrm{~B}$ & 109.0 & $\mathrm{C} 11-\mathrm{C} 12-\mathrm{C} 11$ & $120.8(2)$ \\
\hline $\mathrm{C} 3-\mathrm{C} 4-\mathrm{H} 4 \mathrm{~B}$ & 109.0 & $\mathrm{C} 14-\mathrm{C} 13-\mathrm{C} 12$ & $119.3(3)$ \\
\hline $\mathrm{H} 4 \mathrm{~A}-\mathrm{C} 4-\mathrm{H} 4 \mathrm{~B}$ & 107.8 & $\mathrm{C} 14-\mathrm{C} 13-\mathrm{H} 13$ & 120.3 \\
\hline $\mathrm{C} 6-\mathrm{N} 5-\mathrm{C} 9 \mathrm{~A}$ & $119.3(2)$ & $\mathrm{C} 12-\mathrm{C} 13-\mathrm{H} 13$ & 120.3 \\
\hline $\mathrm{C} 6-\mathrm{N} 5-\mathrm{C} 4$ & $117.9(2)$ & $\mathrm{C} 13-\mathrm{C} 14-\mathrm{C} 15$ & $120.2(3)$ \\
\hline $\mathrm{C} 9 \mathrm{~A}-\mathrm{N} 5-\mathrm{C} 4$ & $122.8(2)$ & $\mathrm{C} 13-\mathrm{C} 14-\mathrm{H} 14$ & 119.9 \\
\hline $\mathrm{N} 6-\mathrm{C} 6-\mathrm{C} 7$ & $122.1(2)$ & $\mathrm{C} 15-\mathrm{C} 14-\mathrm{H} 14$ & 119.9 \\
\hline $\mathrm{N} 6-\mathrm{C} 6-\mathrm{N} 5$ & $116.6(2)$ & $\mathrm{C} 14-\mathrm{C} 15-\mathrm{C} 16$ & $119.2(3)$ \\
\hline $\mathrm{C} 7-\mathrm{C} 6-\mathrm{N} 5$ & $121.2(2)$ & $\mathrm{C} 14-\mathrm{C} 15-\mathrm{H} 15$ & 120.4 \\
\hline $\mathrm{C} 6-\mathrm{N} 6-\mathrm{H} 6 \mathrm{~A}$ & $120(2)$ & $\mathrm{C} 16-\mathrm{C} 15-\mathrm{H} 15$ & 120.4 \\
\hline $\mathrm{C} 6-\mathrm{N} 6-\mathrm{H} 6 \mathrm{~B}$ & $124(2)$ & $\mathrm{C} 15-\mathrm{C} 16-\mathrm{C} 11$ & $123.3(3)$ \\
\hline $\mathrm{H} 6 \mathrm{~A}-\mathrm{N} 6-\mathrm{H} 6 \mathrm{~B}$ & $115(3)$ & $\mathrm{C} 15-\mathrm{C} 16-\mathrm{Cl} 2$ & $116.0(3)$ \\
\hline $\mathrm{C} 6-\mathrm{C} 7-\mathrm{C} 10$ & $119.1(2)$ & $\mathrm{C} 11-\mathrm{C} 16-\mathrm{Cl} 2$ & $120.6(2)$ \\
\hline $\mathrm{C} 6-\mathrm{C} 7-\mathrm{C} 8$ & $123.9(2)$ & $\mathrm{N} 17-\mathrm{C} 17-\mathrm{C} 9$ & $176.9(3)$ \\
\hline $\mathrm{C} 10-\mathrm{C} 7-\mathrm{C} 8$ & $117.0(2)$ & & \\
\hline $\mathrm{C} 9 \mathrm{~A}-\mathrm{N} 1-\mathrm{C} 2-\mathrm{C} 3$ & $46.5(3)$ & $\mathrm{C} 17-\mathrm{C} 9-\mathrm{C} 9 \mathrm{~A}-\mathrm{N} 5$ & $174.5(3)$ \\
\hline $\mathrm{N} 1-\mathrm{C} 2-\mathrm{C} 3-\mathrm{C} 4$ & $-60.3(3)$ & $\mathrm{C} 8-\mathrm{C} 9-\mathrm{C} 9 \mathrm{~A}-\mathrm{N} 5$ & $-1.8(4)$ \\
\hline $\mathrm{C} 2-\mathrm{C} 3-\mathrm{C} 4-\mathrm{N} 5$ & $43.2(3)$ & $\mathrm{C} 6-\mathrm{N} 5-\mathrm{C} 9 \mathrm{~A}-\mathrm{N} 1$ & $170.2(2)$ \\
\hline $\mathrm{C} 3-\mathrm{C} 4-\mathrm{N} 5-\mathrm{C} 6$ & $171.2(2)$ & $\mathrm{C} 4-\mathrm{N} 5-\mathrm{C} 9 \mathrm{~A}-\mathrm{N} 1$ & $-11.5(4)$ \\
\hline $\mathrm{C} 3-\mathrm{C} 4-\mathrm{N} 5-\mathrm{C} 9 \mathrm{~A}$ & $-7.1(4)$ & $\mathrm{C} 6-\mathrm{N} 5-\mathrm{C} 9 \mathrm{~A}-\mathrm{C} 9$ & $-9.1(4)$ \\
\hline $\mathrm{C} 9 \mathrm{~A}-\mathrm{N} 5-\mathrm{C} 6-\mathrm{N} 6$ & $-173.0(2)$ & $\mathrm{C} 4-\mathrm{N} 5-\mathrm{C} 9 \mathrm{~A}-\mathrm{C} 9$ & $169.2(2)$ \\
\hline $\mathrm{C} 4-\mathrm{N} 5-\mathrm{C} 6-\mathrm{N} 6$ & $8.6(4)$ & $\mathrm{C} 7-\mathrm{C} 8-\mathrm{C} 11-\mathrm{C} 12$ & $116.4(3)$ \\
\hline $\mathrm{C} 9 \mathrm{~A}-\mathrm{N} 5-\mathrm{C} 6-\mathrm{C} 7$ & $6.4(4)$ & $\mathrm{C} 9-\mathrm{C} 8-\mathrm{C} 11-\mathrm{C} 12$ & $-118.9(3)$ \\
\hline $\mathrm{C} 4-\mathrm{N} 5-\mathrm{C} 6-\mathrm{C} 7$ & $-172.0(2)$ & $\mathrm{C} 7-\mathrm{C} 8-\mathrm{C} 11-\mathrm{C} 16$ & $-61.5(3)$ \\
\hline $\mathrm{N} 6-\mathrm{C} 6-\mathrm{C} 7-\mathrm{C} 10$ & $4.1(4)$ & $\mathrm{C} 9-\mathrm{C} 8-\mathrm{C} 11-\mathrm{C} 16$ & $63.2(3)$ \\
\hline $\mathrm{N} 5-\mathrm{C} 6-\mathrm{C} 7-\mathrm{C} 10$ & $-175.2(2)$ & $\mathrm{C} 16-\mathrm{C} 11-\mathrm{C} 12-\mathrm{C} 13$ & $1.1(4)$ \\
\hline
\end{tabular}




$\begin{array}{llll}\mathrm{N} 6-\mathrm{C} 6-\mathrm{C} 7-\mathrm{C} 8 & -173.2(2) & \mathrm{C} 8-\mathrm{C} 11-\mathrm{C} 12-\mathrm{C} 13 & -176.9(3) \\ \mathrm{N} 5-\mathrm{C} 6-\mathrm{C} 7-\mathrm{C} 8 & 7.4(4) & \mathrm{C} 16-\mathrm{C} 11-\mathrm{C} 12-\mathrm{C} 11 & -179.0(2) \\ \mathrm{C} 6-\mathrm{C} 7-\mathrm{C} 8-\mathrm{C} 9 & -16.0(3) & \mathrm{C} 8-\mathrm{C} 11-\mathrm{C} 12-\mathrm{C} 11 & 3.0(4) \\ \mathrm{C} 10-\mathrm{C} 7-\mathrm{C} 8-\mathrm{C} 9 & 166.6(2) & \mathrm{C} 11-\mathrm{C} 12-\mathrm{C} 13-\mathrm{C} 14 & -1.0(5) \\ \mathrm{C} 6-\mathrm{C} 7-\mathrm{C} 8-\mathrm{C} 11 & 112.3(3) & \mathrm{C} 11-\mathrm{C} 12-\mathrm{C} 13-\mathrm{C} 14 & 179.1(2) \\ \mathrm{C} 10-\mathrm{C} 7-\mathrm{C} 8-\mathrm{C} 11 & -65.1(3) & \mathrm{C} 12-\mathrm{C} 13-\mathrm{C} 14-\mathrm{C} 15 & 0.5(5) \\ \mathrm{C} 7-\mathrm{C} 8-\mathrm{C} 9-\mathrm{C} 9 \mathrm{~A} & 13.2(3) & \mathrm{C} 13-\mathrm{C} 14-\mathrm{C} 15-\mathrm{C} 16 & -0.2(5) \\ \mathrm{C} 11-\mathrm{C} 8-\mathrm{C} 9-\mathrm{C} 9 A & -113.8(3) & \mathrm{C} 14-\mathrm{C} 15-\mathrm{C} 16-\mathrm{C} 11 & 0.4(4) \\ \mathrm{C} 7-\mathrm{C} 8-\mathrm{C} 9-\mathrm{C} 17 & -163.2(2) & \mathrm{C} 14-\mathrm{C} 15-\mathrm{C} 16-\mathrm{C} 12 & -179.4(2) \\ \mathrm{C} 11-\mathrm{C} 8-\mathrm{C} 9-\mathrm{C} 17 & 69.8(3) & \mathrm{C} 12-\mathrm{C} 11-\mathrm{C} 16-\mathrm{C} 15 & -0.8(4) \\ \mathrm{C} 2-\mathrm{N} 1-\mathrm{C} 9 \mathrm{~A}-\mathrm{C} 9 & 169.2(3) & \mathrm{C} 8-\mathrm{C} 11-\mathrm{C} 16-\mathrm{C} 15 & 177.2(3) \\ \mathrm{C} 2-\mathrm{N} 1-\mathrm{C} 9 \mathrm{~A}-\mathrm{N} 5 & -10.1(4) & \mathrm{C} 12-\mathrm{C} 11-\mathrm{C} 16-\mathrm{Cl} & 179.0(2) \\ \mathrm{C} 17-\mathrm{C} 9-\mathrm{C} 9 \mathrm{~A}-\mathrm{N} 1 & -4.7(4) & \mathrm{C} 8-\mathrm{C} 11-\mathrm{C} 16-\mathrm{C} 12 & -3.1(4) \\ \mathrm{C} 8-\mathrm{C} 9-\mathrm{C} 9 \mathrm{~A}-\mathrm{N} 1 & 179.0(3) & & \end{array}$

Hydrogen-bond geometry $\left(A,{ }^{\circ}\right)$

$\mathrm{Cg} 3$ is the centroid of the $\mathrm{C} 11-\mathrm{C} 16$ dichlorophenyl ring.

\begin{tabular}{lllll}
\hline$D-\mathrm{H} \cdots A$ & $D-\mathrm{H}$ & $\mathrm{H} \cdots A$ & $D \cdots A$ & $D-\mathrm{H} \cdots A$ \\
\hline $\mathrm{N} 1-\mathrm{H} 1 \cdots \mathrm{N} 10^{\mathrm{i}}$ & $0.85(3)$ & $2.43(3)$ & $3.152(3)$ & $143(3)$ \\
$\mathrm{N} 6-\mathrm{H} 6 A \cdots \mathrm{N} 17^{\text {ii }}$ & $0.85(4)$ & $2.17(3)$ & $2.927(3)$ & $149(3)$ \\
$\mathrm{N} 6-\mathrm{H} 6 B \cdots \mathrm{N} 10^{\text {iii }}$ & $0.85(4)$ & $2.16(4)$ & $2.953(3)$ & $156(3)$ \\
$\mathrm{C} 4-\mathrm{H} 4 B \cdots \mathrm{N} 17^{\text {iv }}$ & 0.99 & 2.59 & $3.440(4)$ & 144 \\
$\mathrm{C} 2-\mathrm{H} 2 A \cdots C g 3^{\text {iv }}$ & 0.99 & 2.87 & $3.653(3)$ & 136
\end{tabular}

Symmetry codes: (i) $x+1 / 2,-y+3 / 2, z+1 / 2$; (ii) $x-1 / 2, y-1 / 2, z$; (iii) $x,-y+1, z+1 / 2$; (iv) $x-1 / 2,-y+3 / 2, z+1 / 2$. 